\title{
Development of artificial ribonucleases
}

\author{
Robert Häner*, Jonathan Hall, Angelika Pfützer and Dieter Hüsken
}

Novartis Pharma AG, CH-4002 Basel, Switzerland

\begin{abstract}
Antisense oligonucleotides offer a new way of treating genetically based diseases by interfering with protein expression on the mRNA level. First generation phosphorothioate antisense oligonucleotides have shown promising results in animal experiments and several compounds are currently being tested in the clinics against a variety of diseases. Nevertheless, improvements of various aspects of the technology, such as stability, reduction of length and target affinity of oligonucleotides, are still highly desirable. As a consequence, a large number of chemical modifications of antisense compounds has been reported during the last decade. Unlike phosphorothioates, however, most second generation modifications do not induce degradation of messenger RNA by an RNase H-based mechanism, which is in most cases an essential component of antisense activity. An alternative approach to enable degradation of the mRNA comprises the covalent attachment of RNA cleaving groups to modified oligonucleotides. Macrocyclic lanthanide complexes, in combination with modified antisense oligonucleotides, can be used to specifically cleave mRNA. The preparation, properties and use of such artificial ribonucleases are highlighted. In particular, the design and preparation of constructs cleaving RNA with multiple turn-over is described.
\end{abstract}

\section{INTRODUCTION}

In 1978, Zamecnik and Stephenson (ref. 1) demonstrated that synthetic oligonucleotides inhibited protein synthesis by specific interaction with the messenger RNA (mRNA) coding for the protein. This approach, subsequently called the antisense strategy, triggered considerable interest in oligonucleotides as a new class of potential therapeutic compounds (refs. 2-5). Biological activity of oligonucleotides was then demonstrated in cellular assays and, later, in animals (refs. 6, 7) and hence, several compounds are currently undergoing testing in the clinics against various diseases (ref. 4). Despite the progress made with phosphorothioates (1), improvements in the properties of oligonucleotides with respect to stability, reduction in length and affinity for the RNA target, are still sought (refs. 8, 9). As a consequence, a large number of chemical modifications of antisense compounds has been reported in recent years.

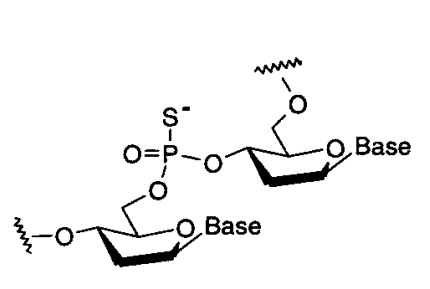

1

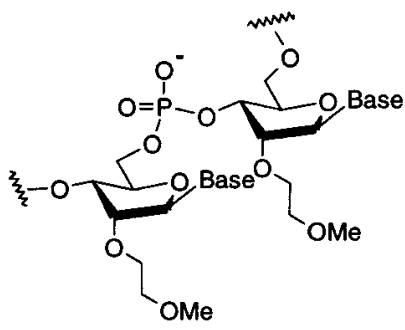

$\underline{2}$

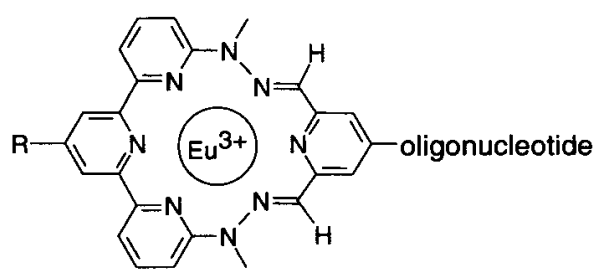

$\underline{3}$

* To whom correspondence should be addressed 
It has been demonstrated that binding of RNase $\mathrm{H}$ to the heteroduplex of a phosphorothioate antisense olignucleotide and its RNA target followed by cleavage of the RNA (illustrated in Scheme 1) is an important component for the activity of antisense oligonucleotides (ref. 10). However, most second generation modifications, such as $2^{\prime}$-methoxyethoxy derivatives $(2$; refs. $9,11,12)$, do not induce degradation of messenger RNA by an RNase $\mathrm{H}$-based mechanism.

Scheme 1: Illustration of RNA cleavage by RNase $\mathrm{H}$ induced by phosphorothioate antisense oligonucleotide.

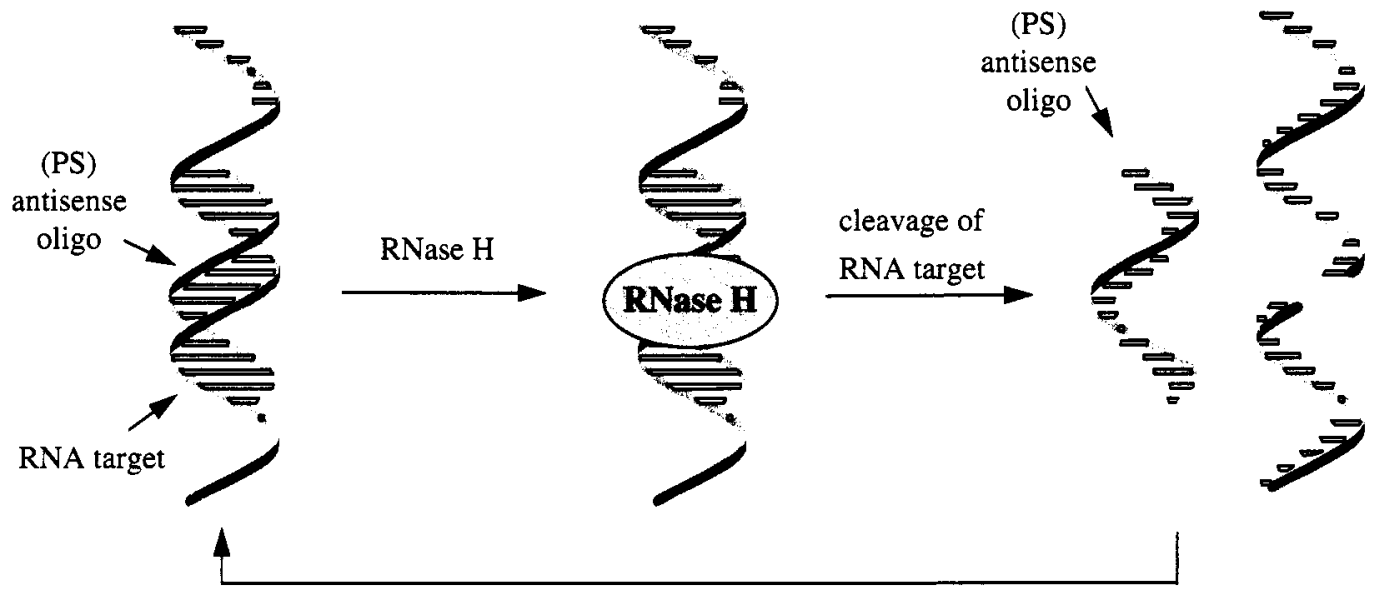

Different approaches were chosen to ensure degradation of mRNA using structurally modified antisense oligonucleotides. One startegy involves the use of chimeric antisense oligonucleotides. Thus, partial modification of the oligonucleotide leads to improvement of binding affinity, stability and some other critical parameters (ref. 8), while the phosphorothioate 'window' still enables induction of RNase $\mathrm{H}$ to cleave the target RNA. Alternatively, we and others investigated a strategy which should allow the destruction of mRNA completely independent of cellular enzymatic activity. Thus, attachment of chemical entities to antisense oligonucleotides capable of hydrolyzing the phosphodiester backbone of RNA, allows the construction of so-called artificial chemical ribonucleases (ref. 13-21). In particular, macrocyclic lanthanide complexes, such as in $\underline{3}$, in combination with modified antisense oligonucleotides, can be used to specifically cleave mRNA. Here, we describe preparation, properties and use of such artificial ribonucleases.

\section{MACROCYCLIC LANTHANIDE COMPLEXES: SYNTHESIS AND CONJUGATION TO OLIGONUCLEOTIDES}

Synthesis of the macrocyclic lanthanide complexes is straightforward and follows the route shown in Scheme 2 (refs. 22, 23). Reaction of the $\alpha, \beta$-unsaturated ketones 4 with $\alpha$-pyridinium-(2-acetyl-6bromopyridine) iodide ( $(\underline{5})$ leads to terpyridines $\underline{6}$ in high $(>90 \%)$ yields. Subsequent treatment with $N$ methylhydrazine leads to the substitution of the two bromide atoms to give $\mathbf{Z}$. Formation of the macrocyclic compounds $\left(\underline{8}, \mathrm{R}^{\prime}=\mathrm{H}\right.$ or methyl) is achieved by treating $\underline{7}$ with 2,6 -diacyl pyridine in the presence of one equivalent of lanthanide (III) salt and a small quantity of conc. hydrochloric acid. The overall procedure for synthesis of the metal complexes ( 4 to $\underline{8}$ ) requires no chromatographic purification steps and is easily scaled up to the kilogram level. Covalent attachment of the metal complexes to oligonucleotides via amide or thiourea linkers is carried out using standard methods. Preparation of 2'-methoxyethoxy modified 
conjugates preferably involves coupling of the metal complex to the solid support-bound oligonucleotides. Due to the remarkable stability of the metal complex, the oligonucleotide conjugates can be deprotected and purified by standard chromatographic or electrophoretic methods without concomitant loss of the metal. They are characterized by MS and capillary electrophoresis.

Scheme 2: General Synthetic Procedure for Preparation of Macrocyclic Lanthanide Complexes.<smiles>O=C(/C=C/c1ccc(Br)cc1)c1cccc(Br)n1</smiles>

4<smiles>O=C(Cn1cccc1)c1cccc(Br)n1</smiles>
5 a)<smiles>Pc1ccc(-c2cc(-c3cccc(Br)n3)nc(-c3cccc(Br)n3)c2)cc1</smiles>

b)<smiles>CN(C)c1cccc(-c2cc(-c3ccc(P)cc3)cc(-c3cccc(N(C)N)n3)n2)n1</smiles>

7

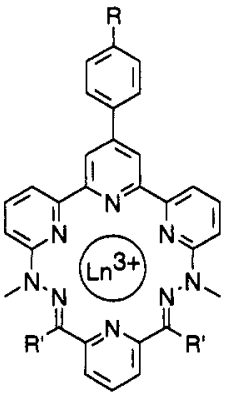

$\underline{8}$

a) $\mathrm{AcOH}, \mathrm{NH}_{4} \mathrm{OAc}$. b) N-Methylhydrazine. c) $\mathrm{LnX}_{3}, \mathrm{HCl}$; 2,6-diacetylpyridine or 2,6-diformylpyridine.

\section{SEQUENCE-SPECIFIC CLEAVAGE OF RNA}

The cleavage activity of the conjugates (e.g. $\underline{9}$ and 10 , Scheme 3) was verified targeting synthetic oligoribonucleotides (refs. 16, 24). Typically, the artificial ribonucleases cleave the target with a half life of $2-4 \mathrm{~h}$ at $37^{\circ} \mathrm{C}(\mathrm{pH} \mathrm{7.4)}$, using an approximate 10 -fold excess of the chemical ribonuclease. Cleavage occurs for the major part in the single stranded region of the RNA strand.

Scheme 3: Autoradiograph of a $12 \%$ denaturing polyacrylamide gel obtained after treatment of a 29-mer oligoribonucleotide $\left({ }^{33} \mathrm{P}\right.$-labelled at the $5^{\prime}$-end; $16 \mathrm{~h}, \mathrm{pH} 7.4,37^{\circ} \mathrm{C}$ ) with conjugates 9 (lane 5) and 10 (lane 6). Lanes 1 and 4: RNA before and after incubation in the reaction buffer; lane 2: alkaline hydrolysis $\left(40 \mathrm{mM} \mathrm{NaOH}, 70^{\circ} \mathrm{C}, 20 \mathrm{~min}\right)$; lane 3: RNase $\mathrm{T} 1$ digestion.

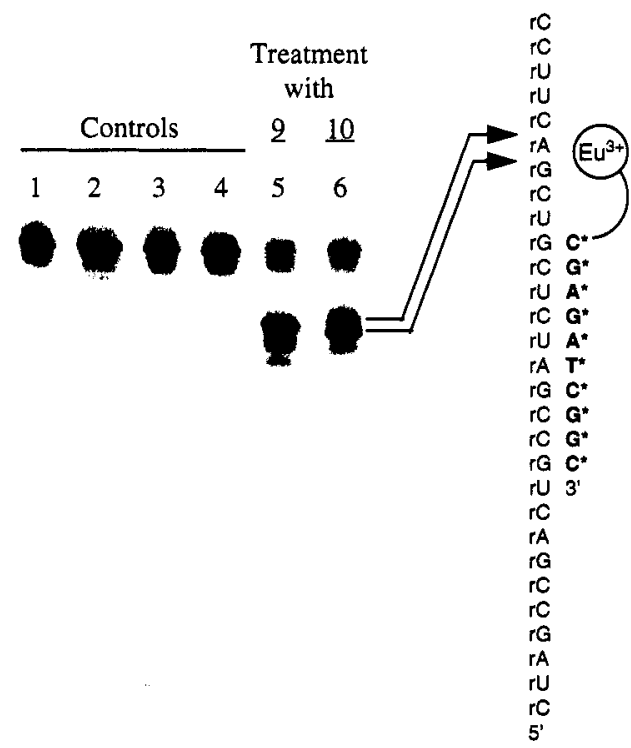

2 Eu-CGAGATCG GCAGTCGGCTAG-3'

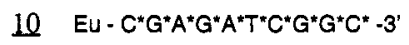

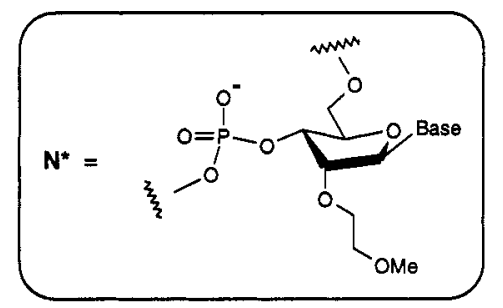


2'-Methoxyethoxy (2'-MOE)-modified cleaver conjugates cleave RNA with equal efficiency as unmodified (deoxy) conjugates, but, due to the increased RNA binding affinity observed with the 2'-MOE modification $\left(\Delta \mathrm{Tm}\right.$ of $+1.1^{\circ} \mathrm{C}$ per modified nucleotide; ref. 9,11$)$, it is possible to use conjugates of much shorter length. Thus, comparable efficiency is observed with the 10mer 2'-MOE modified artificial ribonuclease 10 as with the unmodified $20 \operatorname{mer} \underline{9}$. No cleavage takes place upon treatment of the RNA with an analogous unmodified oligodeoxynucleotide 10 mer conjugate under identical conditions.

\section{ARTIFICIAL CHEMICAL RIBONUCLEASES ACTING WITH MULTIPLE TURN-OVER}

The process of RNA cleavage by oligonucleotide-metal conjugates is summarized in Scheme $4 \mathrm{~A}$. Cleavage is limited to single stranded regions of the RNA. It is known that RNA confined in a helical conformation is much more resistant to metal-catalysed cleavage by transesterification than a corresponding single stranded analog (ref. 25). On the other hand, cleavage of the RNA target in the duplex region should allow artificial ribonucleases to operate with efficient catalytic turnover (ref. 26), as the cleavage event will give two stacked duplexes that would be expected to rapidly dissociate under the reaction conditions. The poor reactivity of ribonucleotides to transesterification/cleavage in the duplex is attributed to the rigid conformation of the phosphodiester backbone, but can be overcome by the introduction of bulges and internal loops in the RNA target strand (ref. 27). To test this hypothesis, artificial nucleases were designed and synthesised comprising lanthanide complexes covalently linked to oligodeoxyribonucleotides which cleave a partially complementary RNA at a bulged ribonucleotide, in the duplex region (ref. 26). Strand scission was found to occur at or near the bulge as illustrated in Scheme $4 B$.

Scheme 4: Ilustration of sequence-specific cleavage of an oligoribonucleotide by an oligonucleotide conjugate in the single strand (A) and at bulged ribonucleotides in the double stranded region (B).

A

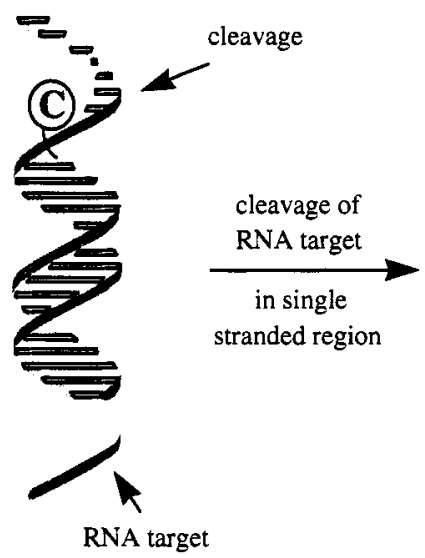

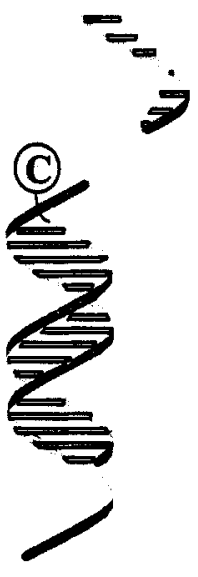

(C) = Lanthanide complex
B

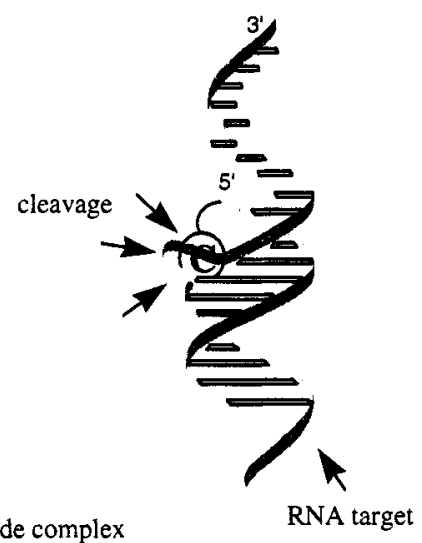

Although turn-over could not be realized via the bulge approach using DNA-cleaver conjugates, multiple turn-over was indeed possible using 2'-MOE conjugates. Several conjugates of various lengths and base composition were tested against bulged RNAs and were observed to cleave more than one equivalent of target RNA. One example is shown in Scheme 5. An excess of oligoribonucleotide 11 is treated at $37^{\circ} \mathrm{C}$ with different ratios of cleaver conjugate $\underline{12}$. Cleavage of 11 takes place at the bulge and, to a lesser extent, also at the 3'-site adjacent to the bulge. Considerably more than one equivalent of RNA is cleaved after 
64h. In the best case, 39 equivalents of RNA (relative to the cleaver conjugate) are cleaved under these conditions. This finding demonstrates that it is possible to design artificial chemical ribonucleases acting in a catalytic way by the bulge approach. Further work is in progress to investigate the kinetics of the cleavage reaction.

Scheme 5: Artificial chemical ribonuclease acting with multiple turnover (conditions: $37^{\circ} \mathrm{C}, 64 \mathrm{hrs}, \mathrm{pH} 7.2$; concentration of 12 is $1 \mu \mathrm{M})$.

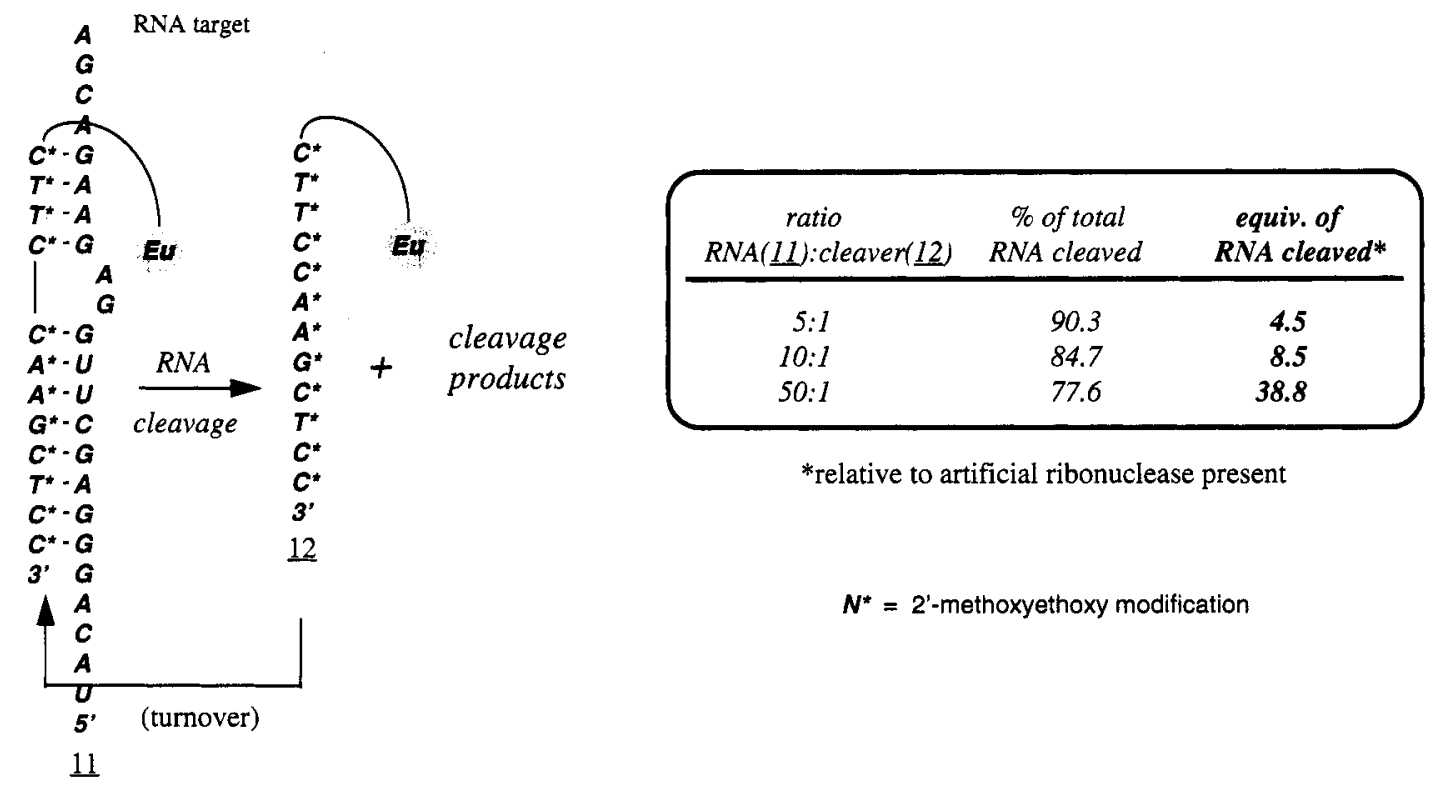

\section{OUTLOOK}

Spurred by potential use in antisense technology, the field of artificial ribonucleases has seen substantial progress over the last five years. While several types of chemical compounds have been linked to oligonucleotides and were shown to cleave RNA in vitro, practical application still remains to be shown. The next step on the way to such practical application is the demonstration of specific RNA cleavage in cellular systems.

\section{REFERENCES}

1. Zamecnik, P.C., Stephenson, M.L. Proc. Natl. Acad. Sci U.S.A. 75, 280 (1978).

2. Agrawal, S. Trends in Biotech. 14, 376 (1996).

3. Matteucci, M.D., and Wagner, R.B. Nature (London), 384 (6604, Suppl.), 20 (1996).

4. Crooke, S.T. Exp. Opin. Invest. Drugs 5, 1047 (1996).

5. Uhlmann, E., and Peyman, A. Chem. Rev. 90, 543 (1990).

6. Akhtar, S., and Agrawal, S. Trends in Pharmacol. Sci. 18, 12 (1997).

7. Monia, B.P., Sasmor, H., Johnston, J.F., Lesnik, E.A., Müller, M., Geiger, T., Altmann, K.-H., Moser, H., and Fabbro, D. Proc. Natl. Acad. Sci. U.S.A., 93, 15481 (1996). 
8. Altmann, K.-H., Dean, N.M., Fabbro, D., Freier, S.M., Geiger, T., Häner, R., Hüsken, D., Martin, P., Monia, B.P., Müller, M., Natt, F., Nicklin, P., Phillips, J., Pieles, U., Sasmor, H., and Moser, H.E. Chimia 50, 168 (1996).

9. DeMesmaeker, A., Häner, R., Martin, P., and Moser, H. Acc. Chem. Res. 28, 366 (1995).

10. Chiang, M., Chan, H., Zounes, M.A., Freier, S.M., Lima, W. and Bennet, C.F. J. Biol. Chem. 266, 18162 (1991).

11. Martin, P. Helv. Chim. Acta 78, 486 (1995).

12. Martin, P. Helv. Chim. Acta 79, 1930 (1996).

13. Häner, R., and Hall, J. Antisense and Nucleic Acids Drug Dev. 7, in press (1997).

14. Bashkin, J.K., Frolova, E.I., and Sampath, U. (1994). J. Am. Chem. Soc. 116, 5981-5982.

15. Magda, D., Miller, R.A., Sessler, J.L., and Iverson, B.L. J. Am. Chem. Soc. 116, 7439 (1994).

16. Hall, J., Hüsken, D., Pieles, U., Moser, H.E., and Häner, R. Chem. \& Biol. 1, 185 (1994).

17. Komiyama, M., and Inokawa, T. J. Biochem. 116, 719 (1994).

18. Matsumura, K., Endo, M., and Komiyama, M. J. Chem. Soc., Chem. Commun., 2019 (1994).

19. Hovinen, J., Guzaev, A., Azhayeva, E., Azhayev, A., and Lönnberg, H. J. Org. Chem. 60, 2205 (1995).

20. Reynolds, M.A., Beck, T.A., Say, P.B., Schwartz, D.A., Dwyer, B.P., Daily, W.J., Vaghefi, M.M., Metzler, M.D., Klem, R.E., and Arnold Jr, L.J. Nucleic Acids Res. 24, 760 (1996).

21. Vlassov, V., Abramova, T., Godovikova, T., Giege, R., and Silnikov, V. Antisense and Nucleic Acid Drug Dev. 7, 39 (1997).

22. Hall, J., Hüsken, D., and Häner, R. Nucleosides \& Nucleotides, in press (1997).

23. Häner, R., Hall, J., and Rihs, G. Helv. Chim. Acta 80, 487 (1997).

24. Häner, R., Hall, J., Hüsken, D., and Moser, H.E. In: DNA Cleavers and Chemotherapy of Cancer and Viral Diseases. (B. Meunier, ed.), pp. 307-320. Nato ASI Series, Ser. C 479; Kluwer Academic Publishers, Netherlands (1996).

25. Kolasa, K. A., Morrow, J.R., and Sharma, A.P. Inorg. Chem. 32, 3983 (1993).

26. Hall, J., Hüsken, D., and Häner, R. Nucleic Acids Res. 24, 3522 (1996).

27. Hüsken, D. Goodall, G., Blommers, M.J.J., Jahnke, W., Hall, J., Häner, R., and Moser, H.E. Biochemistry 35, 16591 (1996). 